bioRxiv preprint doi: https://doi.org/10.1101/2022.01.11.475838; this version posted January 17,2022 . The copyright holder for this preprint (which was not certified by peer review) is the author/funder, who has granted bioRxiv a license to display the preprint in perpetuity. It is made available under aCC-BY 4.0 International license.

\title{
Lightweight compositional analysis of metagenomes with FracMinHash and minimum metagenome covers
}

This manuscript (permalink) was automatically generated from dib-lab/2020-paper-sourmash-gather@b2b79b8 on January $17,2022$.

\section{Authors}

\section{- Luiz Irber}

\section{(iD) 0000-0003-4371-9659 • (?) luizirber · luizirber}

Graduate Group in Computer Science, UC Davis; Department of Population Health and Reproduction, UC Davis .

Funded by Grant GBMF4551 from the Gordon and Betty Moore Foundation; Grant R01HG007513 from the NIH NHGRI

\section{- Phillip T. Brooks}

(ID 0000-0003-3987-244X $\cdot$ (O) brooksph $\cdot$ brooksph

Department of Population Health and Reproduction, UC Davis · Funded by Grant GBMF4551 from the Gordon and Betty Moore Foundation

\section{- Taylor Reiter}

\section{(D) 0000-0002-7388-421X · O taylorreiter · ReiterTaylor}

Graduate Group in Food Science, UC Davis; Department of Population Health and Reproduction, UC Davis · Funded by Grant GBMF4551 from the Gordon and Betty Moore Foundation; Grant R03OD030596 from the NIH Common Fund

\section{- N. Tessa Pierce-Ward}

\section{(iD) 0000-0002-2942-5331 · (D) bluegenes $\cdot y$ saltyscientist}

Department of Population Health and Reproduction, UC Davis · Funded by Grant 1711984 from the NSF; Grant GBMF4551 from the Gordon and Betty Moore Foundation; Grant 2018911 from the NSF

- Mahmudur Rahman Hera

- () mahmudhera - HeraMahmudur

Department of Computer Science and Engineering, Penn State University · Funded by Grant 2029170 from the NSF

\section{- David Koslicki}

\section{(D) 0000-0002-0640-954X · (P dkoslicki · DavidKoslicki}

Department of Computer Science and Engineering, Penn State University; Department of Biology, Penn State University; Huck Institutes of the Life Sciences, Penn State University · Funded by Grant 2029170 from the NSF

\section{- C. Titus Brown}

\section{(D) 0000-0001-6001-2677 · C ctb}

Department of Population Health and Reproduction, UC Davis · Funded by Grant GBMF4551 from the Gordon and Betty Moore Foundation; Grant R01HG007513 from the NIH NHGRI; Grant 2018911 from the NSF; Grant R03OD030596 from the NIH Common Fund 


\section{Abstract}

The identification of reference genomes and taxonomic labels from metagenome data underlies many microbiome studies. Here we describe two algorithms for compositional analysis of metagenome sequencing data. We first investigate the FracMinHash sketching technique, a derivative of modulo hash that supports Jaccard containment estimation between sets of different sizes. We implement FracMinHash in the sourmash software, evaluate its accuracy, and demonstrate large-scale containment searches of metagenomes using 700,000 microbial reference genomes. We next frame shotgun metagenome compositional analysis as the problem of finding a minimum collection of reference genomes that "cover" the known k-mers in a metagenome, a minimum set cover problem. We implement a greedy approximate solution using FracMinHash sketches, and evaluate its accuracy for taxonomic assignment using a CAMI community benchmark. Finally, we show that the minimum metagenome cover can be used to guide the selection of reference genomes for read mapping. sourmash is available as open source software under the BSD 3-Clause license at github.com/diblab/sourmash/.

\section{Introduction}

Shotgun DNA sequencing of microbial communities is an important technique for studying hostassociated and environmental microbiomes [1,2]. By sampling the genomic content of microbial communities, shotgun metagenomics enables the taxonomic and functional characterization of microbiomes $[3,4]$. However, this characterization relies critically on the methods and databases used to interpret the sequencing data $[\underline{5}, \underline{6}, \underline{7}, \underline{8}]$.

Metagenome function and taxonomy are typically inferred from available reference genomes and gene catalogs, via direct genomic alignment [9,10], large-scale protein search [11,12,13], or k-mer matches $[14,15]$. For many of these methods, the substantial increase in the number of available microbial reference genomes (1.1 m in GenBank as of November 2021) presents a significant practical obstacle to comprehensive compositional analyses. Most methods choose representative subsets of available genomic information to analyze; for example, bioBakery 3 provides a database containing $99.2 \mathrm{k}$ reference genomes [9]. Scaling metagenome analysis approaches to make use of the rapidly increasing size of GenBank is an active endeavor in the field $[16,17]$.

Here, we describe a lightweight and scalable approach to compositional analysis of shotgun metagenome data based on finding the minimum set of reference genomes that accounts for all known k-mers in a metagenome - a "minimum metagenome cover". We use a mod-hash based sketching approach for k-mers to reduce memory requirements [18], and implement a polynomialtime greedy approximation algorithm for the minimum set cover analysis [19].

Our approach tackles the selection of appropriate reference genomes for downstream analysis and provides a computationally efficient method for taxonomic classification of metagenome data. Our implementation in the sourmash open source software package works with reference databases containing a million or more microbial genomes and supports multiple taxonomies and private databases.

\section{Results}

We first describe FracMinHash, a sketching technique that supports containment and overlap estimation for DNA sequencing datasets using k-mers. We next frame reference-based metagenome 
content analysis as the problem of finding a minimum set cover for a metagenome using a collection of reference genomes. We then evaluate the accuracy of this approach using a taxonomic classification benchmark. Finally, we demonstrate the utility of this approach by using the genomes from the minimum metagenome cover as reference genomes for read mapping.

\section{FracMinHash sketches support accurate containment operations}

We define the fractional MinHash, or FracMinHash as follows: for a hash function $h: \Omega \rightarrow[O, H]$, on an input set of hash values $W \subseteq \Omega$ and for any $0<=s<=H$,

$$
\operatorname{FRAC}_{s}(W)=\left\{h(w) \leq \frac{H}{s} \mid \forall w \in W\right\}
$$

where $H$ is the largest possible value in the domain of $h(x)$ and $\frac{H}{s}$ is the maximum hash value allowed in the FracMinHash sketch.

The FracMinHash is a mix of MinHash and ModHash $[18,20]$. It keeps the selection of the smallest elements from MinHash, while using the dynamic size from ModHash to allow containment estimation. However, instead of taking $0 \bmod m$ elements like $\mathbf{M O D}_{m}(W)$, a FracMinHash uses the parameter $s$ to select a subset of $W$.

Like ModHash (but not MinHash), FracMinHash supports estimation of the containment index:

$$
\hat{C}_{\text {frac }}(A, B):=\frac{\left|\mathbf{F R A C}_{S}(A) \cap \mathbf{F R A C}_{S}(B)\right|}{\left|\mathbf{F R A C}_{S}(A)\right|}
$$

See Methods for details.

Given a uniform hash function $h$ and $s=m$, the cardinalities of $\mathbf{F R A C} \mathbf{C}_{s}(W)$ and $\mathbf{M O D} \mathbf{D}_{m}(W)$ converge for large $|W|$. The main difference is the range of possible values in the hash space, since the FracMinHash range is contiguous and the ModHash range is not. This permits a variety of convenient operations on the sketches, including iterative downsampling of FracMinHash sketches as well as conversion to MinHash sketches. Beyond accurate containment operations, FracMinHash can be used to estimate evolutionary distance between pairs of sequences undergoing a mutation model, similar to but more accurately than the MinHash derived method in [20]. See [21] for these and other analytical details.

\section{A FracMinHash implementation accurately estimates containment between sets of different sizes}

We compare the FracMinHash method, implemented in the sourmash software [22], to Containment MinHash [23] and Mash Screen (Containment Score) [24] for containment queries in data from the podar mock community, a mock bacterial and archaeal community where the reference genomes are largely known [25]; see also Table 1, row 2. This data set has been used in several methods evaluations $[\underline{24}, \underline{26}, \underline{27}, \underline{28}]$. 
bioRxiv preprint doi: https://doi.org/10.1101/2022.01.11.475838; this version posted January 17,2022 . The copyright holder for this preprint (which was not certified by peer review) is the author/funder, who has granted bioRxiv a license to display the preprint in perpetuity. It is made available under aCC-BY 4.0 International license.

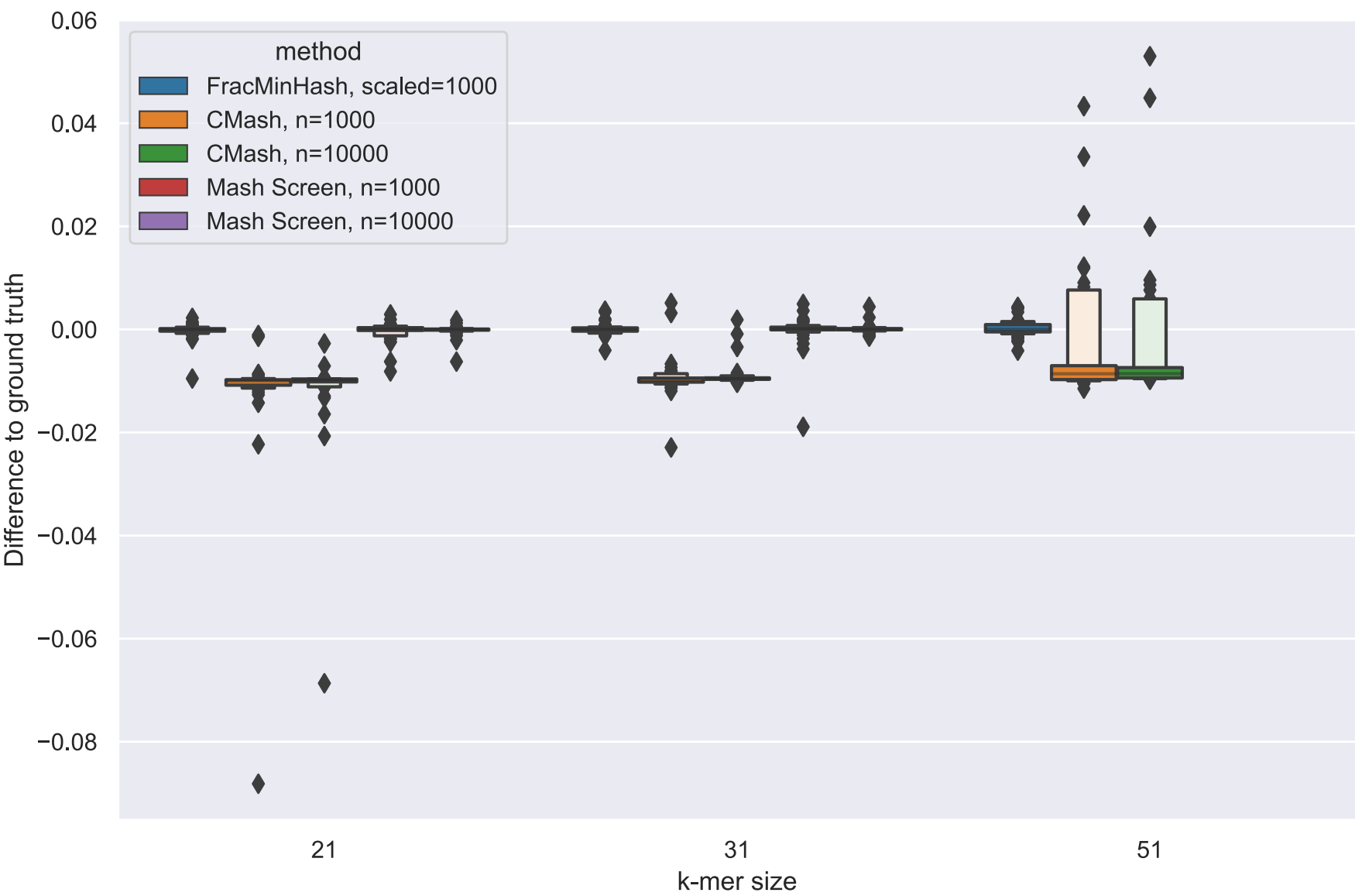

Figure 1: Letter-value plot [29] of the differences from containment estimate to ground truth (exact). Each method is evaluated for $k=\{21,31,51\}$, except for Mash with $k=51$, which is unsupported.

Figure 1 shows containment analysis of genomes in this metagenome, with low-coverage and contaminant genomes (as described in [28] and [24]) removed from the database. All methods are within $1 \%$ of the exact containment on average (Figure 1), with CMash consistently underestimating the containment. Mash Screen with $n=10000$ has the smallest difference to ground truth for $k=\{21,31\}$, followed by FracMinHash with scaled $=100 \odot$ and Mash Screen with $n=1000$. The sourmash sketch sizes varied between 431 hashes and 9540 hashes, with a median of 2741 hashes.

\section{FracMinHash can be used to construct a minimum set cover for metagenomes}

We next ask: what is the smallest collection of genomes in a database that contains all of the known kmers in a metagenome? Formally, for a given metagenome $M$ and a reference database $D$, what is the minimum collection of genomes in $D$ which contain all of the k-mers in the intersection of $D$ and $M$ ? We wish to find the smallest set $\left\{G_{n}\right\}$ of genomes in $D$ such that, for the k-mer decomposition $k()$,

$$
k(M) \cap k(D)=\bigcup_{n}\left\{k(M) \cap k\left(G_{n}\right)\right\}
$$

This is a minimum set covering problem, for which there is a polynomial-time approximation [19]:

1. Initialize $C \leftarrow \emptyset$

2. While $k(M) \cap k(D) \backslash \bigcup_{G \in C}(k(M) \cap k(G))$ is nonempty: 

perpetuity. It is made available under aCC-BY 4.0 International license.
3. $C \leftarrow C \bigcup\left\{\arg \max _{G \in D}\left|k(G) \cup\left(k(M) \cap k(D) \backslash \bigcup_{G \in C}(k(M) \cup k(G))\right)\right|\right\}$
4. return $C$

This greedy algorithm iteratively chooses reference genomes from $D$ in order of largest remaining overlap with $M$, where overlap is in terms of number of k-mers. This results in a progressive classification of the known k-mers in the metagenome to specific genomes. ${ }^{1}$ Note it is classically known that this greedy heuristic results in a logarithmic approximation factor to the optimal set cover solution [19]. This algorithm is implemented as sourmash gather .

In Figure 2, we show an example of this progressive classification of 31-mers by matching GenBank genome for podar mock. The matching genomes are provided in the order found by the greedy algorithm, i.e. by overlap with remaining k-mers in the metagenome. The high rank (early) matches reflect large and/or mostly-covered genomes with high containment, while later matches reflect genomes that share fewer k-mers with the remaining set of k-mers in the metagenome - smaller genomes, less-covered genomes, and/or genomes with substantial overlap with earlier matches. Where there are overlaps between genomes, shared common k-mers are "claimed" by higher rank matches and only k-mer content specific to the later genome is used to find lower rank matches.

As one example of metagenome k-mers shared with multiple matches, genomes from two strains of Shewanella baltica are present in the mock metagenome. These genomes overlap in k-mer content by approximately 50\%, and these shared k-mers are first claimed by Shewanella baltica OS223 - compare S. baltica OS223, rank 8, with S. baltica OS185, rank 33 in Figure 2. Here the difference between the green triangles (all matched k-mers) and red circles (min-set-cov matched k-mers) for S. baltica OS185 represents the k-mers claimed by S. baltica OS223.

For this mock metagenome, $205 \mathrm{~m}$ (54.8\%) of 375m k-mers were found in GenBank (see Table 1, row 2). The remaining $169 \mathrm{~m}$ (45.2\%) k-mers had no matches, and represent either k-mers introduced by sequencing errors or k-mers from real but unknown community members. 
bioRxiv preprint doi: https://doi.org/10.1101/2022.01.11.475838; this version posted January 17,2022 . The copyright holder for this preprint (which was not certified by peer review) is the author/funder, who has granted bioRxiv a license to display the preprint in perpetuity. It is made available under aCC-BY 4.0 International license.
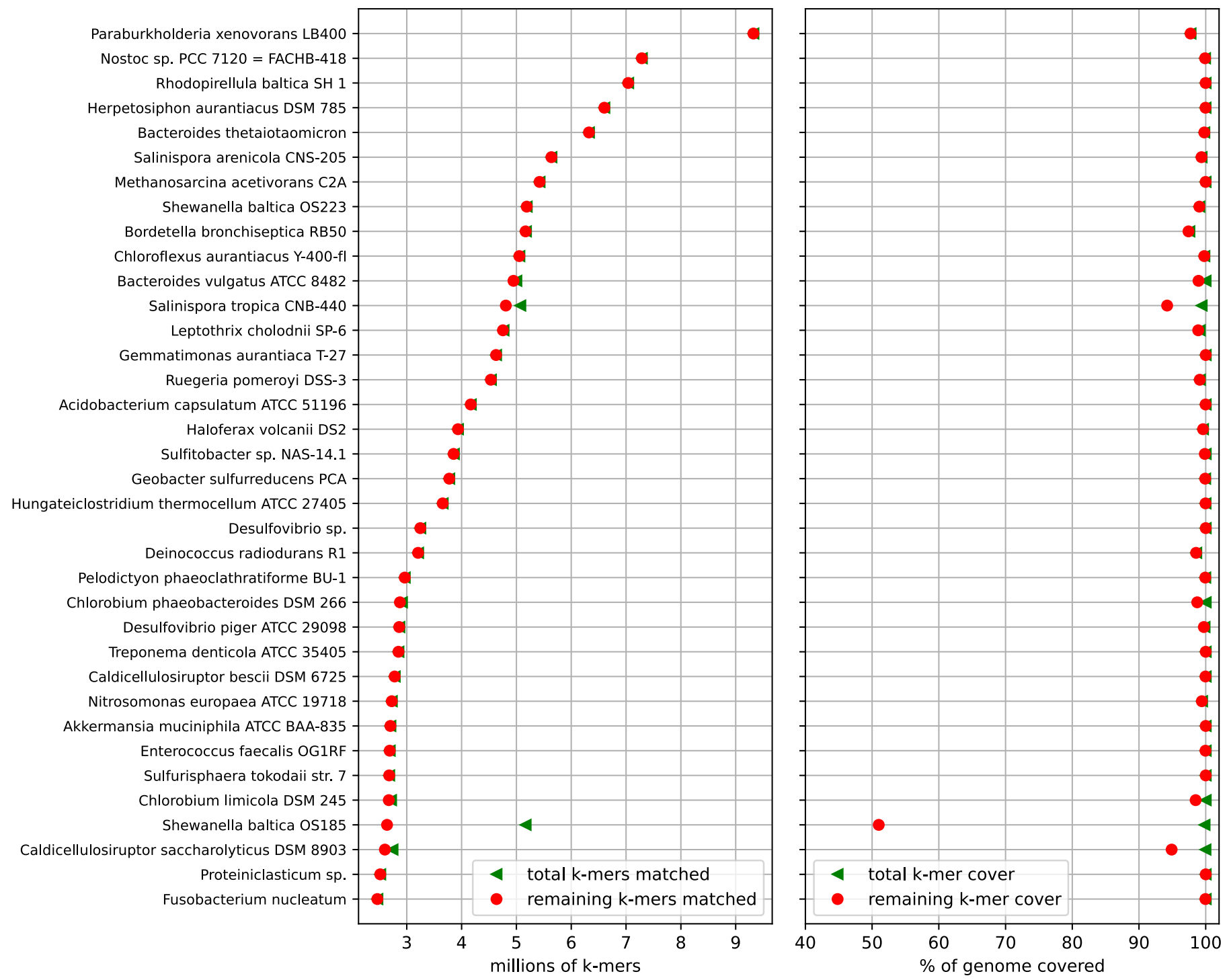

Figure 2: K-mer decomposition of a metagenome into constituent genomes. A rank ordering by remaining containment for the first 36 genomes from the minimum metagenome cover of the podar mock synthetic metagenome [25], calculated using 700,000 genomes from GenBank with scaled=2000, k=31. The $Y$ axis is labeled with the NCBI-designated name of the genome. In the left plot, the $X$ axis represents the estimated number of k-mers shared between each genome and the metagenome. The red circles indicate the number of matching k-mers that were not matched at previous ranks, while the green triangle symbols indicate all matching k-mers. In the right plot, the $X$ axis represents the estimated k-mer coverage of that genome. The red circles indicate the percentage of the genome covered by k-mers remaining at that rank, while the green triangles indicate overlap between the genome and the entire metagenome, including those already assigned at previous ranks.

\section{Minimum metagenome covers can accurately estimate taxonomic composition}

We evaluated the accuracy of min-set-cov for metagenome decomposition using benchmarks from the Critical Assessment of Metagenome Interpretation (CAMI), a community-driven initiative for reproducibly benchmarking metagenomic methods [30]. We used the mouse gut metagenome dataset [31], in which a simulated mouse gut metagenome (MGM) was derived from 791 bacterial and archaeal genomes, representing 8 phyla, 18 classes, 26 orders, 50 families, 157 genera, and 549 species. Sixty-four samples were generated with CAMISIM, with 91.8 genomes present in each sample on average. Each sample is 5 GB in size, and both short-read (Illumina) and long-read (PacBio) simulated sequencing data is available.

Since min-set-cov yields only a collection of genomes, this collection must be converted into a taxonomy with relative abundances for benchmarking with CAMI. We developed the following 
procedure for generating a taxonomic profile from a given metagenome cover. For each genome match, we note the species designation in the NCBI taxonomy for that genome. Then, we calculate the fraction of the genome remaining in the metagenome after k-mers belonging to higher-rank genomes have been removed (i.e. red circles in Figure $\underline{2}$ (a)). We multiply this fraction by the median abundance of the hashes in the sketch to weight the contribution of the genome's species designation to the metagenome taxonomy. This procedure produces an estimate of that species' taxonomic contribution to the metagenome, normalized by the genome size.

a
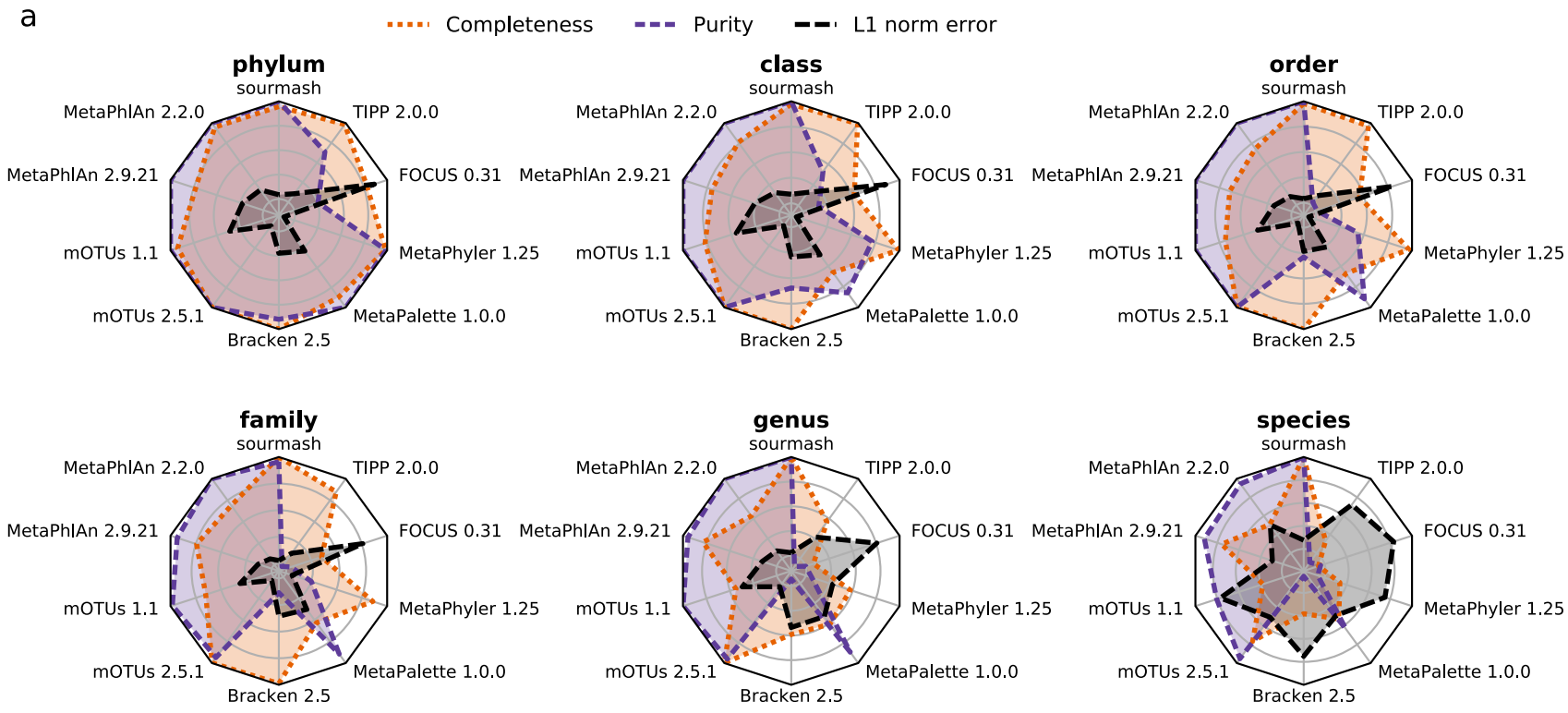

Figure 3: Comparison per taxonomic rank of methods in terms of completeness, purity (1\% filtered), and L1 norm.

\begin{tabular}{|c|c|c|c|c|}
\hline C & Completeness & Purity (1\% filtered) & L1 norm error & Sum of scores \\
\hline 1st & sourmash (247) & sourmash (179) & mOTUs 2.5 .1 (789) & sourmash (1262) \\
\hline $2 \mathrm{nd}$ & mOTUs 2.5 .1 (416) & MetaPhIAn 2.2 .0 (241) & sourmash (836) & mOTUs 2.5 .1 (1887) \\
\hline $3 r d$ & Bracken 2.5 (1008) & mOTUs 1.1 (631) & MetaPhIAn 2.9.21 (1401) & MetaPhIAn 2.2 .0 (3527) \\
\hline 4th & MetaPhyler 1.25 (1298) & mOTUs 2.5 .1 (682) & MetaPhIAn 2.2 .0 (1497) & MetaPhIAn 2.9.21 (4349) \\
\hline 5 th & TIPP 2.0 .0 (1424) & MetaPhIAn 2.9.21 (789) & MetaPhyler 1.25 (1586) & MetaPhyler 1.25 (5148) \\
\hline 6 th & MetaPhIAn 2.2 .0 (1789) & MetaPalette 1.0 .0 (1182) & mOTUs 1.1 (2317) & mOTUs 1.1 (5253) \\
\hline 7 th & MetaPhIAn 2.9.21 (2159) & MetaPhyler 1.25 (2264) & TIPP 2.0 .0 (2361) & MetaPalette 1.0 .0 (5989) \\
\hline 8th & mOTUs 1.1 (2305) & Bracken 2.5 (2881) & MetaPalette 1.0 .0 (2390) & Bracken 2.5 (6574) \\
\hline 9th & MetaPalette 1.0 .0 (2417) & TIPP 2.0 .0 (3361) & Bracken 2.5 (2685) & TIPP 2.0 .0 (7146) \\
\hline 10th & FOCUS 0.31 (3424) & FOCUS 0.31 (3764) & FOCUS 0.31 (3894) & FOCUS 0.31 (11082) \\
\hline
\end{tabular}

Figure 4: Methods rankings and scores obtained for the different metrics over all samples and taxonomic ranks. For score calculation, all metrics were weighted equally. A scaled value of 2000 and a k-mer size of 31 was used.

In Figures $\underline{3}$ and $\underline{4}$ we show an updated version of Figure 6 from [31] that includes our method, implemented in the sourmash software and benchmarked using OPAL [32]. The minimum metagenome cover was calculated against the Jan 8, 2019 snapshot of RefSeq provided by the CAMI project. Here we compare 10 different methods for taxonomic profiling and their characteristics at each taxonomic rank. While previous methods show reduced completeness - the ratio of taxa correctly identified in the ground truth - below the genus level, sourmash can reach $88.7 \%$ completeness at the species level with the highest purity (the ratio of correctly predicted taxa over all predicted taxa) across all methods: $95.9 \%$ when filtering predictions below $1 \%$ abundance, and $97 \%$ for unfiltered results. sourmash also has the second lowest L1-norm error, the highest number of true positives and the lowest number of false positives. 


\section{Minimum metagenome covers select small subsets of large databases}

Table 1: Four metagenomes and the number of genomes in the estimated minimum metagenome cover from GenBank, with scaled=2000 and k=31. Overlap and \% 31-mers identified are estimated from FracMinHash sketch size.

\begin{tabular}{|l|l|l|l|}
\hline \multicolumn{1}{|c|}{ data set } & \multicolumn{1}{|c|}{$\begin{array}{c}\text { genomes }>\text { = 100k 31-mer } \\
\text { overlap }\end{array}$} & size of min-set-cov & \% 31-mers identified \\
\hline zymo mock & 405,839 & 19 & $47.1 \%$ \\
\hline podar mock & 5,800 & 74 & $54.8 \%$ \\
\hline gut real & 96,423 & 99 & $36.0 \%$ \\
\hline oil well real & 1,235 & 135 & $14.9 \%$ \\
\hline
\end{tabular}

In Table 1, we show the minimum metagenome cover for four metagenomes against GenBank - two mock communities [25,33], a human gut microbiome data set from iHMP [3], and an oil well sample [34]. Our implementation provides estimates for both the total number of genomes with substantial overlap to a query genome, and the minimum set of genomes that account for k-mers with overlap in the query metagenome. Note that only matches estimated to have more than 100,000 overlapping kmers are shown (see Methods for details).

We find many genomes with overlaps for each metagenome, due to the redundancy of the reference database. For example, zymo mock contains a Salmonella genome, and there are over 200,000 Salmonella genomes that match to it in GenBank. Likewise, gut real matches to over 75,000 E. coli genomes in GenBank. Since neither podar mock nor oil well real contain genomes from species with substantial representation in GenBank, they yield many fewer total overlapping genomes.

Regardless of the number of genomes in the database with substantial overlap, the estimated minimum collection of genomes is always much smaller than the number of genomes with overlaps. In the cases where the k-mers in the metagenome are mostly identified, this is because of database redundancy: e.g. in the case of zymo mock, the min-set-cov algorithm chooses precisely one Salmonella genome from the 200,000+ available. Conversely, in the case of oil well real, much of the sample is not identified, suggesting that the small size of the covering set is because much of the sample is not represented in the database.

\section{Minimum metagenome covers provide representative genomes for mapping}

Mapping metagenome reads to representative genomes is an important step in many microbiome analysis pipelines, but mapping approaches struggle with large, redundant databases [16,17]. One specific use for a minimum metagenome cover could be to select a small set of representative genomes for mapping. We therefore developed a hybrid selection and mapping pipeline that uses the rank-ordered min-set-cov results to map reads to candidate genomes.

We first map all metagenome reads to the first ranked genome in the minimum metagenome cover, and then remove successfully mapped reads from the metagenome. Remaining unmapped reads are then mapped to the second rank genome, and this then continues until all genomes have been used. That is, all reads mapped to the rank- 1 genome in Figure 2 are removed from the rank- 2 genome mapping, and all reads mapping to rank- 1 and rank- 2 genomes are removed from the rank-3 genome 
bioRxiv preprint doi: https://doi.org/10.1101/2022.01.11.475838; this version posted January 17,2022 . The copyright holder for this preprint (which was not certified by peer review) is the author/funder, who has granted bioRxiv a license to display the preprint in perpetuity. It is made available under aCC-BY 4.0 International license.

mapping, and so on. This produces results directly analogous to those presented in Figure 2 , but for reads rather than k-mers. This approach is implemented in the automated workflow package genome-grist; see Methods for details.

Figure $\underline{5}$ compares k-mer assignment rates and mapping rates for the four evaluation metagenomes in Table 1. Broadly speaking, we see that k-mer-based estimates of metagenome composition agree closely with the number of bases covered by mapped reads: the $Y$ axis has not been re-scaled, so kmer matches and read mapping coverage correspond well. This suggests that the k-mer-based minset-cov approach effectively selects reference genomes for metagenome read mapping.

For mock metagenomes (Figure $\underline{5}(A)$ and $(B)$ ), there is a close correspondence between mapping and k-mer coverage, while for real metagenomes (Figure $\underline{5}(C)$ and (D)), mapping coverage tends to be higher. This may be because the mock metagenomes are largely constructed from strains with known genomes, so most 31-mers match exactly, while the gut and oil well metagenomes contain a number of strains where only species (and not strain) genomes are present in the database, and so mapping performs better. Further work is needed to evaluate rates of variation across a larger number of metagenomes.
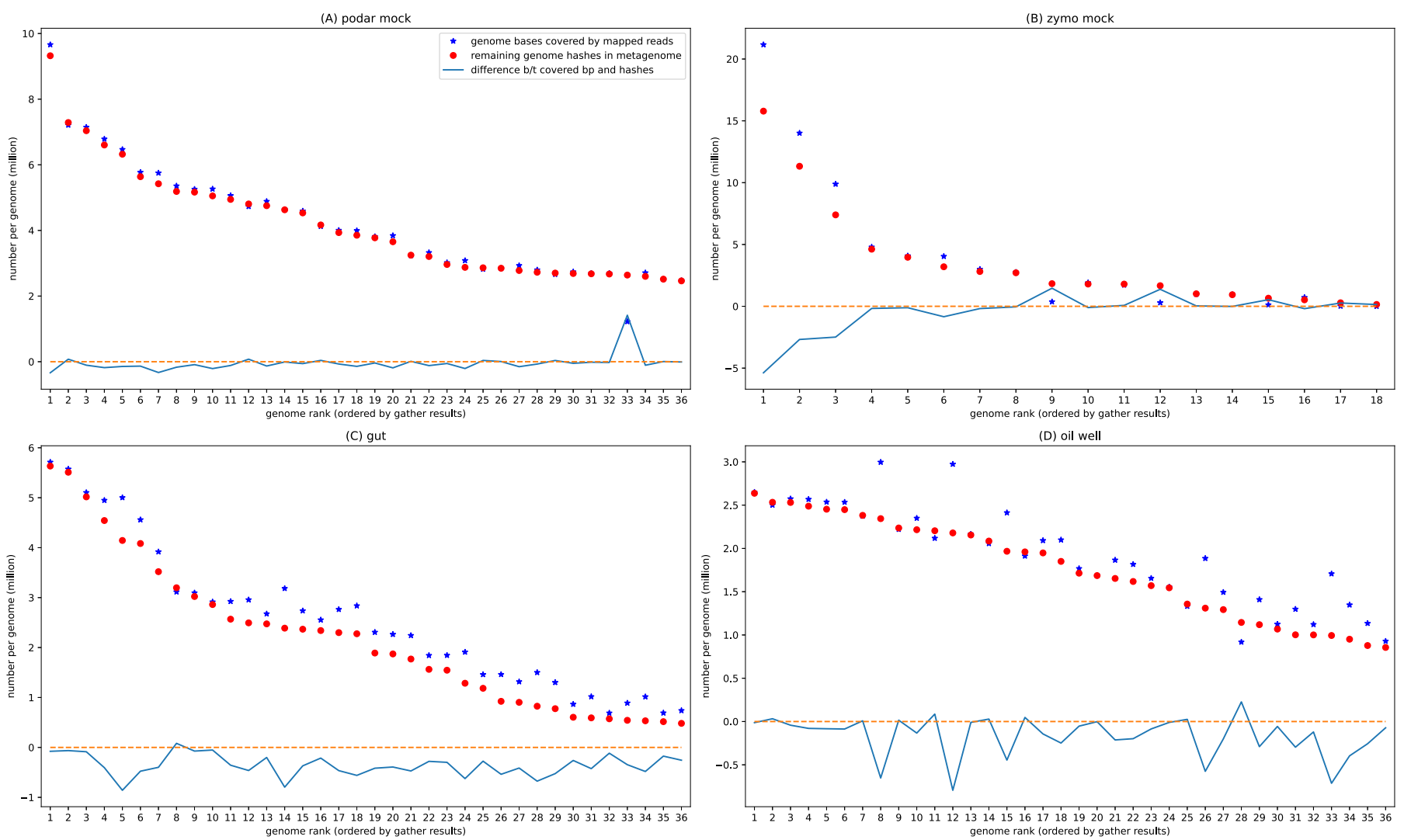

Figure 5: Hash-based k-mer decomposition of a metagenome into constituent genomes compares well to bases covered by read mapping. Plots for each of four metagenomes showing estimated k-mer overlap per genome, along with bases covered by read mapping, for the first 36 genomes in the minimum metagenome cover. The reference genomes are rank ordered along the $X$ axis (as in the $Y$ axis for Figure 2), based on the largest number of hashes from the metagenome specific to that genome; hence the number of hashes classified for each genome (red circles) is monotonically decreasing. The $\mathrm{Y}$ axis shows estimated number of k-mers classified to this genome (red circles) or total number of bases in the reference covered by mapped reads (blue stars); the numbers have not been rescaled. Decreases in mapping (peaks in blue lines) occur for genomes which are not exact matches to the genomes of the organisms used to build the mock community; for example, in (A), the peak at rank 33 of podar mock is for S. baltica OS185, and represents reads that were preferentially mapped to S. baltica OS223, rank 8.

\section{Discussion}




\section{FracMinHash provides efficient containment queries for large data sets.}

FracMinHash is a derivative of ModHash that uses the bottom hashing concept from MinHash to support containment operations: all elements in the set to be sketched are hashed, and any hash values below a certain fixed boundary value are kept for the sketch. This fixed boundary value is determined by the desired accuracy for the sketch operations, with clear space/time constraint tradeoffs.

Intuitively, FracMinHash can be viewed as performing density sampling at a rate of $1 k$-mer per $s$ distinct k-mers seen, where $s$ is used to define a boundary value $\frac{H}{s}$ for the bottom sketch.

FracMinHash can also be viewed as a type of lossy compression, with a fixed compression ratio of $s$ : for values of $s$ used here ( $s \approx 1000)$, k-mer sets are reduced in cardinality by 1000-fold.

Unlike MinHash, FracMinHash supports containment estimation between sets of very different sizes, and here we demonstrate that it can be used efficiently and effectively for compositional analysis of shotgun metagenome data sets with k-mers. In particular, FracMinHash is competitive in accuracy with extant MinHash-based techniques for containment analysis, while also supporting Jaccard similarity. In addition, FracMinHash can be used to obtain point estimates of and confidence intervals around mutation rates and evolutionary distances; see [21] for these and other analytical results.

We note that the FracMinHash technique has been used under a number of different names, including Scaled MinHash [35,36], universe minimizers [37], Shasta markers [38], and mincode syncmers [39]. The name FracMinHash was coined by Kristoffer Sahlin in an online discussion on Twitter [40] and chosen by discussants as the least ambiguous option. We use it here accordingly.

FracMinHash offers several conveniences over MinHash. No hash is ever removed from a FracMinHash sketch during construction; thus sketches grow proportionally to the number of distinct k-mers in the sampled data set, but also support many operations - including all of the operations used here - without needing to revisit the original data set. This is in contrast to MinHash, which requires auxiliary data structures for many operations - most especially, containment operations $[23,24]$. Thus FracMinHash sketches serve as compressed indices for the original content for a much broader range of operations than MinHash.

Because FracMinHash sketches collect all hash values below a fixed threshold, they also support streaming analysis of sketches: any operations that used a previously selected value can be cached and updated with newly arriving values. ModHash has similar properties, but this is not the case for MinHash: after $n$ values are selected any displacement caused by new data can invalidate previous calculations.

FracMinHash also directly supports the addition and subtraction of hash values from a sketch, allowing for limited types of post-processing and filtering without revisiting the original data set. This includes unions and intersections. Although possible for MinHash, in practice this requires oversampling (using a larger $n$ ) to account for possibly having fewer than $n$ values after filtering, e.g. see the approach taken in Finch [41].

When the multiplicity of hashes in the original data is retained, FracMinHash sketches can be filtered on abundance. This allows removing low-abundance values, as implemented in Finch [41]. Filtering 
bioRxiv preprint doi: https://doi.org/10.1101/2022.01.11.475838; this version posted January $17,2022$. The copyright holder for this preprint (which was not certified by peer review) is the author/funder, who has granted bioRxiv a license to display the preprint in perpetuity. It is made available under aCC-BY 4.0 International license.

values that only appear once was implemented in Mash by using a Bloom filter and only adding values after they were seen once; later versions also implemented an extra counter array to keep track of counts for each value in the MinHash. These operations can be done in FracMinHash without auxiliary data structures.

Another useful operation available on FracMinHash sketches is downsampling. the contiguous value range for FracMinHash sketches means that MinHash sketches can be extracted from FracMinHash sketches whenever the size of the requested MinHash is less than the size of the FracMinHash sketch. Likewise, MinHash sketches can be losslessly converted to FracMinHash sketches when the maximum hash value in the MinHash sketch is larger than $H / s$.

Finally, because FracMinHash sketches are simply collections of hashes, existing hash-based k-mer indexing approaches can be applied to sketches to support fast search with both similarity and containment estimators; several index types, including Sequence Bloom Trees [42] and reverse indices, are provided in the sourmash software.

In exchange for these many conveniences, FracMinHash sketches have limited sensitivity for small data sets where the k-mer cardinality of the data set $\approx s$, and are only bounded in size by $H / s$, which is typically quite large $\approx 2 e 16$. The limited sensitivity of sketches may affect the sensitivity of geneand viral genome-sized queries, but at $s=1000$ we see comparable accuracy and sketch size to MinHash for bacterial genome comparisons (Figure 1).

\section{Minimum set covers can be used for accurate compositional analysis of metagenomes.}

Many metagenome content analysis approaches use reference genomes to interpret the metagenome content, but most such approaches rely on starting with a list of reduced-redundancy genomes from a much larger database (e.g. bioBakery 3 selects approximately 100,000 genomes [9]), which can reduce sensitivity and precision [17]. Here, we incorporate this reduction into the overall workflow by searching the complete database for a minimum set of reference genomes necessary to account for all k-mers shared between the metagenome and the database. We show that this can be resolved efficiently for real-world data sets; implementing a greedy min-set-cov approximation algorithm on top of FracMinHash, we provide an approach that readily scales to 700,000 genomes on current hardware. We show that in practice this procedure reduces the number of genomes under consideration to $\approx 100$ for several mock and real metagenomes.

The development of a small list of relevant genomes is particularly useful for large reference databases containing many redundant genomes; for example, in Table 1 , we show that for one mock and one real community, we select minimum metagenome covers of 19 and 99 genomes for metagenomes that contain matches to $406 \mathrm{k}$ and $96 \mathrm{k}$ GenBank genomes total.

The min-set-cov approach for assigning genomes to metagenomes using k-mers differs substantially from extant k-mer and mapping-based approaches for identifying relevant genomes. LCA-based approaches such as Kraken label individual k-mers based on taxonomic lineages in a database, and then use the resulting database of annotated k-mers to assign taxonomy to reads. Mapping- and homology-based approaches such as Diamond use read mapping to genomes or read alignment to gene sequences in order to assign taxonomy and function [43]. These approaches typically focus on assigning individual k-mers or reads. In contrast, here we analyze the entire collection of k-mers and assign them in aggregate to the best genome match, and then repeat until no matches remain. 
bioRxiv preprint doi: https://doi.org/10.1101/2022.01.11.475838; this version posted January $17,2022$. The copyright holder for this preprint (which was not certified by peer review) is the author/funder, who has granted bioRxiv a license to display the preprint in perpetuity. It is made available under aCC-BY 4.0 International license.

The resulting minimum metagenome cover can then be used as part of further analyses, including both taxonomic content analysis and read mapping. For taxonomic analyses, we find that this approach is competitive with other current approaches and has several additional conveniences (discussed in detail below). The comparison of hash-based estimation of containment to mapping results in Figure $\underline{5}$ suggests that this approach is an accurate proxy for systematic mapping, as also seen in Metalign [17].

There is one significant drawback to assigning minimum metagenome covers based on k-mers: because k-mers are not a perfect proxy for mapping (e.g. see Figure $\underline{5}$, blue lines), using k-mers to identify the best genome for mapping may sometimes lead to inaccurate assignments. Note that long k-mers are generally more stringent and specific than mapping, so e.g. 51-mer overlaps can be used to identify some candidate genomes for mapping, but not all candidate genomes will necessarily be found using 51-mer overlaps. The extent and impact of this kind of false negative in the min-set-cov approach remains to be evaluated but is likely to only affect strain- and species-level assignments, since nucleotide similarity measures lose sensitivity across more distant taxonomic ranks [44].

Our implementation of the min-set-cov algorithm in sourmash also readily supports using custom reference databases as well as updating minimum metagenome covers with the addition of new reference genomes. When updating metagenome covers with new reference genomes, the first stage of calculating overlaps can be updated with the new genomes (column 2 of Table 1), while the actual calculation of a minimum set cover must be redone each time.

Minimum set cover approaches may provide opportunities beyond those discussed here. For example, read- and contig-based analyses, and analysis and presentation of alignments, can be potentially simplified with this approach.

\section{Minimum metagenome covers support accurate and flexible taxonomic assignment}

We can build a taxonomic classifier on top of minimum metagenome covers by reporting the taxonomies of the constituent genomes, weighted by distinct overlap and aggregated at the relevant taxonomic levels. Our CAMI-based taxonomic benchmarking shows that this approach is competitive with several extant approaches for all metrics across all taxonomic levels (Figures $\underline{3}$ and $\underline{4}$ ). This taxonomic accuracy also suggests that minimum metagenome covers themselves are likely to be accurate, since the taxonomic assignment is built solely on the metagenome cover.

One convenient feature of this approach to taxonomic analysis is that new or changed taxonomies can be readily incorporated by assigning them directly to genome identifiers; the majority of the computational work here is involved in finding the reference genomes, which can have assignments in multiple taxonomic frameworks. For example, sourmash already supports GTDB [45] natively, and will also support the emerging LINS framework [46]. sourmash can also readily incorporate updates to taxonomies, e.g. the frequent updates to the NCBI taxonomy, without requiring expensive reanalysis of the primary metagenome data or even regenerating the minimum metagenome cover.

Interestingly, this framing of taxonomic classification as a minimum set cover problem may also avoid the loss of taxonomic resolution that affects k-mer- and read-based approaches on large databases [47]; this is because we incorporate taxonomy after reads and k-mers have been assigned to individual genomes, and choose entire genomes based on a greedy best-match-first approach. This minimizes the impact of individual k-mers that may be common to a genus or family, or were misassigned as a result of contamination. 
bioRxiv preprint doi: https://doi.org/10.1101/2022.01.11.475838; this version posted January 17,2022 . The copyright holder for this preprint (which was not certified by peer review) is the author/funder, who has granted bioRxiv a license to display the preprint in perpetuity. It is made available under aCC-BY 4.0 International license.

Finally, as the underlying min-set-cov implementation supports custom databases, it is straightforward to support taxonomic analysis using custom databases and/or custom taxonomic assignments. This is potentially useful for projects that are generating many new genomes and wish to use them for metagenome analysis. sourmash natively supports this functionality.

Our current implementation of taxonomic assignment in sourmash does not provide read-level assignment. However, it is a straightforward (if computationally expensive) exercise to use the read mapping approach developed in this paper to provide read-level taxonomic assignment along with genome abundance estimation.

\section{The minimum set cover approach is reference dependent}

The min-set-cov approach is reference-based, and hence is entirely dependent on the reference database. This may present challenges: for example, in many cases the exact reference strains present in the metagenome will not be present in the database. This manifests in two ways - see Figure $\underline{5}$. First, for real metagenomes, there is a systematic mismatch between the hash content and the mapping content (green line), because mapping software is more permissive in the face of variants than k-mer-based exact matching. Moreover, many of the lower rank genomes in the plot are from the same species but different strains as the higher ranked genomes, suggesting that strainspecific portions of the reference are being utilized for matching at lower ranks. In reality, there will usually be a different mixture of strains in the metagenome than is present in the reference database. Methods for updating references from metagenome data sets may provide an opportunity for generating metagenome-specific references [48].

The approach presented here chooses arbitrarily between matches with equivalent numbers of contained k-mers. There are specific genomic circumstances where this approach could usefully be refined with additional criteria. For example, if a phage genome is present in the reference database, and is also present within one or more genomes in the database, it may desirable to select the match with the highest Jaccard similarity in order to choose the phage genome. This is algorithmically straightforward to implement when desired.

In light of the strong reference dependence of the min-set-cov approach together with the insensitivity of the FracMinHash technique, it may be useful to explore alternate methods of summarizing the list of overlapping genomes, that is, summarizing all the genomes in column 2 of Table 1. For example, a hierarchical approach could be taken to first identify the full list of overlapping genomes using FracMinHash at a low resolution, followed by a higher resolution (but more resource intensive) approach to identify the best matching genomes.

\section{Opportunities for future improvement of min-set-cov}

There are a number of immediate opportunities for future improvement of the min-set-cov approach.

Implementing min-set-cov on top of FracMinHash means our approach may incorrectly choose between very closely related genomes, because the set of subsampled hashes may not discriminate between them. Likewise, the potentially very large size of the sketches may inhibit the application of this approach to very large metagenomes.

These limitations are not intrinsic to min-set-cov, however; any data structure supporting both the containment $C(A, B)=\frac{|A \cap B|}{|A|}$ and remove elements operations can be used to implement the greedy approximation algorithm. For example, a simple set of the $k$-mer composition of the query supports element removal, and calculating containment can be done with regular set operations. 
Approximate membership query (AMQ) sketches like the Counting Quotient Filter [49] can also be used, with the benefit of reduced storage and memory usage.

In turn, this means that limitations of our current implementation, such as insensitivity to small genomes when $s$ is approximately the same as the genome size, may be readily solvable with other sketch types.

There are other opportunities for improving on these initial explorations. The availability of abundance counts for each element in the FracMinHash is not well explored, since the process of removing elements from the query does not use them. This may be important for genomes with more repetitive content such as eukaryotic genomes. Both the multiple match as well as the abundance counts issues can benefit from existing solutions taken by other methods, like the species score (for disambiguation) and Expectation-Maximization (for abundance analysis) approaches from Centrifuge [50].

\section{Conclusion}

The FracMinHash and min-set-cov approaches explored here provide powerful and accurate techniques for analyzing metagenomes, with well defined limitations. We show several immediate applications for both taxonomic and mapping-based analysis of metagenomes. We provide an implementation of these approaches in robust open-source software, together with workflows to enable their practical use on large data sets. The approaches also offer many opportunities for further exploration and improvement with different data structures, alternative approximation algorithms, and additional summarization approaches.

\section{Methods}

\section{Analytical analysis of FracMinHash}

Given two arbitrary sets $A$ and $B$ which are subsets of a domain $\Omega$, the containment index $C(A, B)$ is defined as $C(A, B):=\frac{|A \cap B|}{|A|}$. Let $h$ be a perfect hash function $h: \Omega \rightarrow[0, H]$ for some $H \in \mathbb{R}$. For a scale factor $s$ where $0 \leq s \leq 1$, a FracMinHash sketch of a set $A$ is defined as follows:

$$
\text { FRAC } \mathbf{C}_{S}(A)=\{h(a) \mid \forall a \in A \text { s.t. } h(a) \leq H s\}
$$

The scale factor $s$ is a tunable parameter that can modify the size of the sketch. Using this FracMinHash sketch, we define the FracMinHash estimate of the containment index $\hat{C}_{\text {frac }}(A, B)$ as follows:

$$
\hat{C}_{\text {frac }}(A, B):=\frac{\left|\mathbf{F R A C} \mathbf{C}_{S}(A) \cap \mathbf{F R A C}_{S}(B)\right|}{\left|\mathbf{F R A C} \mathbf{C}_{S}(A)\right|}
$$

For notational simplicity, we define $X_{A}:=\left|\mathbf{F R A C} \mathbf{C}_{S}(A)\right|$. Observe that if one views $h$ as a uniformly distributed random variable, we have that $X_{A}$ is distributed as a binomial random variable: $X_{A} \sim \operatorname{Binom}(|A|, s)$. Furthermore, if $A \cap B \neq \emptyset$ where both $A$ and $B$ are non-empty sets, then $X_{A}$ and $X_{B}$ are independent when the probability of success is strictly smaller than 1 . Using these notations, we compute the expectation of $\hat{C}_{\text {frac }}(A, B)$. 
Theorem 1: For $0<s<1$, if $A$ and $B$ are two distinct sets such that $A \cap B$ is non-empty,

$$
\mathrm{E}\left[\hat{C}_{\text {frac }}(A, B) \mathbb{1}_{\left|\mathbf{F R A C}_{S}(A)\right|>0}\right]=\frac{|A \cap B|}{|A|}\left(1-(1-s)^{|A|}\right) .
$$

Proof. Using the notation introduced previously, observe that

$$
\hat{C}_{\text {frac }}(A, B) \mathbb{1}_{\left|\mathbf{F R A C}_{S}(A)\right|>0}=\frac{X_{A \cap B}}{X_{A \cap B}+X_{A \backslash B}} \mathbb{1}_{X_{A \cap B}+X_{A \backslash B}>0},
$$

and that the random variables $X_{A \cap B}$ and $X_{A \backslash B}$ are independent (which follows directly from the fact that $A \cap B$ is non-empty, and because $A$ and $B$ are distinct, $A \backslash B$ is also non-empty). We will use the following fact from standard calculus:

$$
\int_{0}^{1} x t^{x+y-1} d t=\frac{x}{x+y} \mathbb{1}_{x+y>0}
$$

Then using the moment generating function of the binomial distribution, we have

$$
\begin{aligned}
& \mathrm{E}\left[t_{A \cap B}^{X}\right]=(1-s+s t)^{|A \cap B|} \\
& \mathrm{E}\left[t_{A \backslash B}^{X}\right]=(1-s+s t)^{|A \backslash B|} .
\end{aligned}
$$

We also know by continuity that

$$
\begin{aligned}
\mathrm{E}\left[X_{A \cap B} t^{X_{A \cap B}-1}\right] & =\frac{d}{d t}(1-s+s t)^{|A \cap B|} \\
& =|A \cap B| s(1-s+s t)^{|A \cap B|-1} .
\end{aligned}
$$

Using these observations, we can then finally calculate that

$$
\begin{aligned}
\mathrm{E}\left[\frac{X_{A \cap B}}{X_{A \cap B}+X_{A \backslash B}} \mathbb{1}_{X_{A \cap B}+X_{A \backslash B}>0},\right] & =\mathrm{E}\left[\int_{0}^{1} X_{A \cap B} t^{X_{A \cap B}+X_{A \backslash B}-1} d t\right] \\
& =\int_{0}^{1} \mathrm{E}\left[X_{A \cap B} t^{X_{A \cap B}+X_{A \backslash B}-1} d t\right] \\
& =\int_{0}^{1} \mathrm{E}\left[X_{A \cap B} t^{X_{A \cap B}-1}\right] \mathrm{E}\left[t_{A \backslash B}^{X}\right] d t \\
& =|A \cap B| \int_{0}^{1}(1-s+s t)^{|A \cap B|+|A \backslash B|-1} d t \\
& =\left.\frac{|A \cap B|(1-s+s t)^{|A|}}{|A|}\right|_{t=0} ^{t=1} \\
& =\frac{|A \cap B|}{|A|}\left(1-(1-s)^{|A|}\right),
\end{aligned}
$$

using Fubini's theorem and independence. 
In light of Theorem 1, we note that $\hat{C}_{\text {frac }}(A, B)$ is not an unbiased estimate of $C(A, B)$. This may explain the observations in [36] that show suboptimal performance for short sequences (e.g. viruses). However, for sufficiently large $|A|$ and $s$, the bias factor $\left(1-(1-s)^{|A|}\right)$ is sufficiently close to 1 .

Hence we can define:

$$
C_{\text {frac }}(A, B)=\frac{|A \cap B|}{|A|}\left(1-(1-s)^{|A|}\right)^{-1}
$$

which will have expectation

by Theorem 1.

$$
\mathrm{E}\left[C_{\text {frac }}(A, B)\right]=\frac{|A \cap B|}{|A|}
$$

\section{Implementation of FracMinHash and min-set-cov}

We provide implementations of FracMinHash and min-set-cov in the software package sourmash, which is implemented in Python and Rust and developed under the BSD license [22]. FracMinHash sketches were created for DNA sequence inputs using the sourmash sketch dna command with the scaled parameter. Minimum metagenome covers were generated using sourmash gather with the sketched metagenome as query against a collection of one or more sketched genomes.

sourmash is available at github.com/sourmash-bio/sourmash. The results in this paper were generated with sourmash v4.2.3.

\section{Comparison between CMash, mash screen, and Scaled MinHash.}

Experiments use $k=\{21,31,51\}$ (except for Mash, which only supports $k \leq 32$ ). For Mash and CMash they were run with $n=\{1000,10000\}$ to evaluate the containment estimates when using larger sketches with sizes comparable to the FracMinHash sketches with scaled $=1000$. The truth set is calculated using an exact $k$-mer counter implemented with a HashSet data structure in the Rust programming language [51]. The sourmash results were generated with sourmash search -containment .

For Mash Screen the ratio of hashes matched by total hashes is used instead of the Containment Score, since the latter uses a $k$-mer survival process modeled as a Poisson process first introduced in [52] and later used in the Mash distance [20] and Containment score [24] formulations.

\section{GenBank database sketching and searches}

Minimum metagenome covers were calculated using a microbial genome subset of GenBank (July 2020, 725,339 genomes) using a scaled factor of 2000 and a k-mer size of 31. Sketches for all genomes and metagenomes were calculated with sourmash sketch dna $-p$ scaled $=2000, k=31$. The minimum metagenome covers were calculated using all genomes sharing 50 hashes with the metagenome (that is, an estimated overlap of 100,000 k-mers) with sourmash gather -threshold-bp 1e5. Overlapping sketches were saved with --save-prefetch and matches were saved with --save-matches. 
bioRxiv preprint doi: https://doi.org/10.1101/2022.01.11.475838; this version posted January 17,2022 . The copyright holder for this preprint (which was not certified by peer review) is the author/funder, who has granted bioRxiv a license to display the preprint in perpetuity. It is made available under aCC-BY 4.0 International license.

The GenBank database used is $24 \mathrm{~GB}$ in size and is available for download through the sourmash project [53].

\section{Taxonomy}

The CAMI evaluations were run with the sourmash CAMI pipeline [54] against the Jan 8, 2019 RefSeq snapshot provided by CAMI. This pipeline generated Open-community Profiling Assessment (OPAL) compatible output [30]. This output was then processed with the standard CAMI tools.

\section{Read mapping and hybrid mapping pipeline}

Metagenome reads were mapped to reference genomes using minimap2 v2.17 [55] with short singleend read mapping mode ( $-x$ sr ).

The hybrid selection and mapping pipeline using the rank-ordered min-set-cov results was implemented in the subtract_gather.py script in the genome-grist package [56].

The complete workflow, from metagenome download to taxonomic analysis and iterative mapping, is implemented in the genome-grist package. genome-grist uses snakemake [57] to define and execute a workflow that combines sourmash sketching, metagenome cover calculation, and taxonomic analysis with metagenome download from the SRA, genome download from GenBank, and read mapping. We used genome-grist v0.7.4 [58] to generate the results in this paper; see conf-paper.yml in the pipeline repository.

genome-grist relies on matplotlib [59], Jupyter Notebook [60], numpy [61], pandas [62], papermill, samtools [63], bedtools [64], fastp [65], khmer [66], screed [67], seqtk [68], and sra-tools [69]. These tools are all installed and managed in snakemake via conda [70] and bioconda [71]. genome-grist itself is developed under the BSD 3-clause open source license, and is available at github.com/dib-

lab/genome-grist/.

\section{Intermediate data products and figure generation}

All figures were generated using the Jupyter Notebooks from v0.1 of the github.com/dib-lab/2021paper-sourmash-gather-pipeline repository [72]. This repository also contains the intermediate data products necessary for figure generation.

\section{Metagenome data set accessions}

The accessions for the metagenome data sets in Table 1 are:

\begin{tabular}{|l|l|}
\hline \multicolumn{1}{|c|}{ data set } & \multicolumn{1}{c|}{ SRA accession } \\
\hline zymo mock & SRR12324253 \\
\hline podar mock & SRR606249 \\
\hline gut real & SRR5650070 \\
\hline oil well real & SRR1976948 \\
\hline
\end{tabular}




\section{References}

1. A genomic catalog of Earth's microbiomes

Stephen Nayfach, Simon Roux, Rekha Seshadri, Daniel Udwary, Neha Varghese, Frederik Schulz, Dongying Wu, David Paez-Espino, I-Min Chen, Marcel Huntemann, ... IMG/M Data Consortium Nature Biotechnology (2020-11-09) https://doi.org/ghjh4b

DOI: $10.1038 / \mathrm{s} 41587-020-0718-6 \cdot P M I D: \underline{33169036} \cdot P M C I D: \underline{\text { PMC8041624 }}$

2. Metagenomic assessment of the global diversity and distribution of bacteria and fungi Mohammad Bahram, Tarquin Netherway, Clémence Frioux, Pamela Ferretti, Luis Pedro Coelho, Stefan Geisen, Peer Bork, Falk Hildebrand Environmental Microbiology (2020-12-02) https://doi.org/gjcw9f DOI: 10.1111/1462-2920.15314 · PMID: $\underline{33185929}$ · PMCID: PMC7898879

3. The Integrative Human Microbiome Project Nature (2019-05) https://doi.org/gf3wpg DOI: 10.1038/s41586-019-1238-8 · PMID: 31142853 ·PMCID: PMC6784865

4. Structure and function of the global ocean microbiome

Shinichi Sunagawa, Luis Pedro Coelho, Samuel Chaffron, Jens Roat Kultima, Karine Labadie, Guillem Salazar, Bardya Djahanschiri, Georg Zeller, Daniel R Mende, Adriana Alberti, ... Science (2015-05-22) https://doi.org/4tr

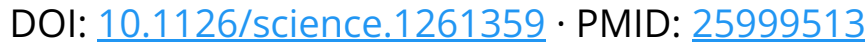

5. Priorities for the next $\mathbf{1 0}$ years of human microbiome research

Lita Proctor

Nature (2019-05-29) https://doi.org/gnnprk

DOI: $10.1038 / d 41586-019-01654-0 \cdot P M I D: \underline{31142863}$

6. Challenges in benchmarking metagenomic profilers

Zheng Sun, Shi Huang, Meng Zhang, Qiyun Zhu, Niina Haiminen, Anna Paola Carrieri, Yoshiki Vázquez-Baeza, Laxmi Parida, Ho-Cheol Kim, Rob Knight, Yang-Yu Liu

Nature Methods (2021-05-13) https://doi.org/gj2n7w

DOI: 10.1038/s41592-021-01141-3 ·PMID: 33986544 ·PMCID: PMC8184642

7. Critical Assessment of Metagenome Interpretation - the second round of challenges

F Meyer, A Fritz, Z-L Deng, D Koslicki, A Gurevich, G Robertson, M Alser, D Antipov, F Beghini, D Bertrand, ... AC McHardy

Cold Spring Harbor Laboratory (2021-07-12) https://doi.org/gk566x

DOI: $10.1101 / 2021.07 .12 .451567$

8. A review of methods and databases for metagenomic classification and assembly

Florian P Breitwieser, Jennifer Lu, Steven L Salzberg

Briefings in Bioinformatics (2019-07) https://doi.org/gdq95k

DOI: $10.1093 / \mathrm{bib} / \mathrm{bbx120} \cdot$ PMID: $29028872 \cdot P M C I D:$ PMC6781581

9. Integrating taxonomic, functional, and strain-level profiling of diverse microbial communities with bioBakery 3

Francesco Beghini, Lauren J Mclver, Aitor Blanco-Míguez, Leonard Dubois, Francesco Asnicar, Sagun Maharjan, Ana Mailyan, Paolo Manghi, Matthias Scholz, Andrew Maltez Thomas, ... Nicola Segata

eLife (2021-05-04) https://doi.org/gkc38n

DOI: $10.7554 /$ elife.65088 · PMID: 33944776 ·PMCID: PMC8096432 
bioRxiv preprint doi: https://doi.org/10.1101/2022.01.11.475838; this version posted January 17,2022 . The copyright holder for this preprint (which was not certified by peer review) is the author/funder, who has granted bioRxiv a license to display the preprint in perpetuity. It is made available under aCC-BY 4.0 International license.

10. MEGAN-LR: new algorithms allow accurate binning and easy interactive exploration of metagenomic long reads and contigs

Daniel H Huson, Benjamin Albrecht, Caner Bağcı, Irina Bessarab, Anna Górska, Dino Jolic, Rohan BH Williams

Biology Direct (2018-01) https://doi.org/gnnprp

DOI: $10.1186 /$ s13062-018-0208-7 ·PMID: 29678199 · PMCID: PMC5910613

11. Fast and sensitive taxonomic assignment to metagenomic contigs

M Mirdita, M Steinegger, F Breitwieser, J Söding, E Levy Karin

Bioinformatics (2021-09-15) https://doi.org/gnnprm

DOI: 10.1093/bioinformatics/btab184 · PMID: 33734313 ·PMCID: PMC8479651

12. Microbial abundance, activity and population genomic profiling with mOTUs2

Alessio Milanese, Daniel R Mende, Lucas Paoli, Guillem Salazar, Hans-Joachim Ruscheweyh,

Miguelangel Cuenca, Pascal Hingamp, Renato Alves, Paul I Costea, Luis Pedro Coelho, ... Shinichi

Sunagawa

Nature Communications (2019-03-04) https://doi.org/gfwktp

DOI: 10.1038/s41467-019-08844-4 ·PMID: $\underline{30833550} \cdot$ PMCID: $\underline{\text { PMC6399450 }}$

13. eggNOG 5.0: a hierarchical, functionally and phylogenetically annotated orthology resource based on $\mathbf{5 0 9 0}$ organisms and 2502 viruses

Jaime Huerta-Cepas, Damian Szklarczyk, Davide Heller, Ana Hernández-Plaza, Sofia K Forslund, Helen Cook, Daniel R Mende, Ivica Letunic, Thomas Rattei, Lars J Jensen, ... Peer Bork

Nucleic Acids Research (2018-11-12) https://doi.org/gg8bdg

DOI: $10.1093 /$ nar/gky1085 · PMID: 30418610 · PMCID: PMC6324079

14. Improved metagenomic analysis with Kraken 2

Derrick E Wood, Jennifer Lu, Ben Langmead

Genome Biology (2019-11-28) https://doi.org/ggfk55

DOI: 10.1186/s13059-019-1891-0 · PMID: 31779668 · PMCID: PMC6883579

15. Fast and sensitive taxonomic classification for metagenomics with Kaiju

Peter Menzel, Kim Lee Ng, Anders Krogh

Nature Communications (2016-04-13) https://doi.org/f8h4b6

DOI: $10.1038 /$ ncomms11257 · PMID: $27071849 \cdot$ PMCID: PMC4833860

16. ganon: precise metagenomics classification against large and up-to-date sets of reference sequences

Vitor C Piro, Temesgen H Dadi, Enrico Seiler, Knut Reinert, Bernhard Y Renard

Bioinformatics (2020-07) https://doi.org/gnxxz8

DOI: 10.1093/bioinformatics/btaa458 · PMID: 32657362 ·PMCID: PMC7355301

17. Metalign: efficient alignment-based metagenomic profiling via containment min hash

Nathan LaPierre, Mohammed Alser, Eleazar Eskin, David Koslicki, Serghei Mangul

Genome Biology (2020-09-10) https://doi.org/ghtgrz

DOI: 10.1186/s13059-020-02159-0 · PMID: $\underline{32912225}$ ·PMCID: PMC7488264

18. On the resemblance and containment of documents

AZ Broder

Institute of Electrical and Electronics Engineers (IEEE) (2002-11-22) https://doi.org/fqk7hr

DOI: $10.1109 /$ sequen.1997.666900

19. A Greedy Heuristic for the Set-Covering Problem

V Chvatal

Mathematics of Operations Research (1979-08) https://doi.org/dx2gd2 
bioRxiv preprint doi: https://doi.org/10.1101/2022.01.11.475838; this version posted January $17,2022$. The copyright holder for this preprint (which was not certified by peer review) is the author/funder, who has granted bioRxiv a license to display the preprint in perpetuity. It is made available under aCC-BY 4.0 International license.

DOI: $10.1287 /$ moor.4.3.233

20. Mash: fast genome and metagenome distance estimation using MinHash

Brian D Ondov, Todd J Treangen, Páll Melsted, Adam B Mallonee, Nicholas H Bergman, Sergey

Koren, Adam M Phillippy

Genome Biology (2016-06-20) https://doi.org/gfx74q

DOI: 10.1186/s13059-016-0997-X ·PMID: 27323842 · PMCID: PMC4915045

21. Debiasing FracMinHash and deriving confidence intervals for mutation rates across a wide range of evolutionary distances

Mahmudur Rahman Hera, NTessa Pierce-Ward, David Koslicki

Cold Spring Harbor Laboratory (2022-01-14) https://doi.org/gn342h

DOI: $10.1101 / 2022.01 .11 .475870$

22. sourmash: a library for MinHash sketching of DNA

C Titus Brown, Luiz Irber

The Journal of Open Source Software (2016-09-14) https://doi.org/ghdrk5

DOI: $10.21105 /$ joss.00027

23. IMPROVING MIN HASH VIA THE CONTAINMENT INDEX WITH APPLICATIONS TO

METAGENOMIC ANALYSIS

David Koslicki, Hooman Zabeti

Cold Spring Harbor Laboratory (2017-09-04) https://doi.org/ghvn6z

DOI: $10.1101 / 184150$

24. Mash Screen: high-throughput sequence containment estimation for genome discovery

Brian D Ondov, Gabriel J Starrett, Anna Sappington, Aleksandra Kostic, Sergey Koren,

Christopher B Buck, Adam M Phillippy

Genome Biology (2019-11-05) https://doi.org/ghtgmb

DOI: 10.1186/s13059-019-1841-x ·PMID: $\underline{31690338}$ ·PMCID: PMC6833257

25. Comparative metagenomic and rRNA microbial diversity characterization using archaeal and bacterial synthetic communities

Migun Shakya, Christopher Quince, James H Campbell, Zamin K Yang, Christopher W Schadt, Mircea Podar

Environmental Microbiology (2013-06) https://doi.org/f42ccr

DOI: 10.1111/1462-2920.12086 · PMID: 23387867 · PMCID: PMC3665634

26. Omega: an Overlap-graph de novo Assembler for Metagenomics

Bahlul Haider, Tae-Hyuk Ahn, Brian Bushnell, Juanjuan Chai, Alex Copeland, Chongle Pan

Bioinformatics (2014-10) https://doi.org/f6kt42

DOI: 10.1093/bioinformatics/btu395 · PMID: 24947750

27. metaSPAdes: a new versatile metagenomic assembler

Sergey Nurk, Dmitry Meleshko, Anton Korobeynikov, Pavel A Pevzner

Genome Research (2017-05) https://doi.org/f97jkv

DOI: 10.1101/gr.213959.116 · PMID: 28298430 · PMCID: PMC5411777

28. Evaluating Metagenome Assembly on a Simple Defined Community with Many Strain

Variants

Sherine Awad, Luiz Irber, CTitus Brown

Cold Spring Harbor Laboratory (2017-06-25) https://doi.org/ghvn6x

DOI: $\underline{10.1101 / 155358}$

29. Letter-Value Plots: Boxplots for Large Data

Heike Hofmann, Hadley Wickham, Karen Kafadar 
bioRxiv preprint doi: https://doi.org/10.1101/2022.01.11.475838; this version posted January $17,2022$. The copyright holder for this preprint (which was not certified by peer review) is the author/funder, who has granted bioRxiv a license to display the preprint in perpetuity. It is made available under aCC-BY 4.0 International license.

Journal of Computational and Graphical Statistics (2017-07-03) https://doi.org/gf38v7 DOI: $10.1080 / 10618600.2017 .1305277$

30. Critical Assessment of Metagenome Interpretation-a benchmark of metagenomics software

Alexander Sczyrba, Peter Hofmann, Peter Belmann, David Koslicki, Stefan Janssen, Johannes Dröge, Ivan Gregor, Stephan Majda, Jessika Fiedler, Eik Dahms, ... Alice C McHardy

Nature Methods (2017-10-02) https://doi.org/gbzspt

DOI: $\underline{10.1038 / n m e t h .4458} \cdot$ PMID: 28967888 · PMCID: PMC5903868

31. Tutorial: assessing metagenomics software with the CAMI benchmarking toolkit

Fernando Meyer, Till-Robin Lesker, David Koslicki, Adrian Fritz, Alexey Gurevich, Aaron E Darling, Alexander Sczyrba, Andreas Bremges, Alice C McHardy

Nature Protocols (2021-03-01) https://doi.org/gh77rh

DOI: $10.1038 / 541596-020-00480-3 \cdot P M I D: \underline{33649565}$

32. Assessing taxonomic metagenome profilers with OPAL

Fernando Meyer, Andreas Bremges, Peter Belmann, Stefan Janssen, Alice C McHardy, David

Koslicki

Genome Biology (2019-03-04) https://doi.org/gf9vbw

DOI: 10.1186/s13059-019-1646-y. PMID: 30832730 ·PMCID: PMC6398228

33. ZymoBIOMICS Microbial Community Standards

ZYMO RESEARCH

https://www.zymoresearch.com/collections/zymobiomics-microbial-community-standards

34. Genome-Resolved Metagenomic Analysis Reveals Roles for Candidate Phyla and Other Microbial Community Members in Biogeochemical Transformations in Oil Reservoirs Ping Hu, Lauren Tom, Andrea Singh, Brian C Thomas, Brett J Baker, Yvette M Piceno, Gary L Andersen, Jillian F Banfield mBio (2016-03-02) https://doi.org/f8j5xr

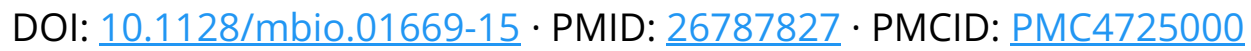

35. Large-scale sequence comparisons with sourmash

NTessa Pierce, Luiz Irber, Taylor Reiter, Phillip Brooks, CTitus Brown

F1000Research (2019-07-04) https://doi.org/gf9v84

DOI: 10.12688/f1000research.19675.1 · PMID: $\underline{31508216}$ •PMCID: PMC6720031

36. luizirber/phd 2020.09.28

Luiz Irber, CTitus Brown, Gabriel Marcondes

Zenodo (2020-09-28) https://zenodo.org/record/4057151

37. Minimizer-space de Bruijn graphs: Whole-genome assembly of long reads in minutes on a personal computer

Barış Ekim, Bonnie Berger, Rayan Chikhi

Cell Systems (2021-10) https://doi.org/gmtsjc

DOI: 10.1016/j.cels.2021.08.009 · PMID: $\underline{34525345}$ · PMCID: PMC8562525

38. Nanopore sequencing and the Shasta toolkit enable efficient de novo assembly of eleven human genomes

Kishwar Shafin, Trevor Pesout, Ryan Lorig-Roach, Marina Haukness, Hugh E Olsen, Colleen Bosworth, Joel Armstrong, Kristof Tigyi, Nicholas Maurer, Sergey Koren, ... Benedict Paten

Nature Biotechnology (2020-05-04) https://doi.org/ggvsn4

DOI: 10.1038/s41587-020-0503-6 · PMID: 32686750 ·PMCID: PMC7483855 
39. Syncmers are more sensitive than minimizers for selecting conserved k-mers in biological sequences

Robert Edgar

PeerJ (2021-02-05) https://doi.org/gm7pzp

DOI: $10.7717 /$ peerj.10805 · PMID: $33604186 \cdot P M C I D:$ PMC7869670

40. https://twitter.com/krsahlin/status/1463169988689285125

Twitter

https://twitter.com/krsahlin/status/1463169988689285125

41. Finch: a tool adding dynamic abundance filtering to genomic MinHashing

Roderick Bovee, Nick Greenfield

The Journal of Open Source Software (2018-02-01) https://doi.org/gm85dx

DOI: $10.21105 / j$ joss.00505

42. Fast search of thousands of short-read sequencing experiments

Brad Solomon, Carl Kingsford

Nature Biotechnology (2016-02-08) https://doi.org/f8ddk3

DOI: 10.1038/nbt.3442 · PMID: 26854477 · PMCID: PMC4804353

43. DIAMOND+MEGAN: Fast and Easy Taxonomic and Functional Analysis of Short and Long Microbiome Sequences

Caner Bağcl, Sascha Patz, Daniel H Huson

Current Protocols (2021-03-03) https://doi.org/gjhfck

DOI: $\underline{10.1002 / c p z 1.59} \cdot$ PMID: $\underline{33656283}$

44. MetaPalette: a k -mer Painting Approach for Metagenomic Taxonomic Profiling and

Quantification of Novel Strain Variation

David Koslicki, Daniel Falush

mSystems (2016-06-28) https://doi.org/gg3gㅁ

DOI: 10.1128/msystems.00020-16 · PMID: 27822531 · PMCID: PMC5069763

45. GTDB: an ongoing census of bacterial and archaeal diversity through a phylogenetically consistent, rank normalized and complete genome-based taxonomy

Donovan H Parks, Maria Chuvochina, Christian Rinke, Aaron J Mussig, Pierre-Alain Chaumeil, Philip Hugenholtz

Nucleic Acids Research (2022-01-07) https://doi.org/gm97d8

DOI: 10.1093/nar/gkab776 · PMID: 34520557 PMCID: PMC8728215

46. A Proposal for a Genome Similarity-Based Taxonomy for Plant-Pathogenic Bacteria that Is Sufficiently Precise to Reflect Phylogeny, Host Range, and Outbreak Affiliation Applied to $<\mathrm{i}>$ Pseudomonas syringae sensu lato $</ \mathrm{i}>$ as a Proof of Concept

Boris A Vinatzer, Alexandra J Weisberg, Caroline L Monteil, Haitham A Elmarakeby, Samuel K Sheppard, Lenwood S Heath

Phytopathology® (2017-01) https://doi.org/gg78hd

DOI: 10.1094/phyto-07-16-0252-r·PMID: 27552324

47. RefSeq database growth influences the accuracy of k-mer-based lowest common ancestor species identification

Daniel J Nasko, Sergey Koren, Adam M Phillippy, Todd J Treangen

Genome Biology (2018-10-30) https://doi.org/ggc9db

DOI: $10.1186 /$ s13059-018-1554-6 ·PMID: 30373669 · PMCID: PMC6206640

48. Exploring neighborhoods in large metagenome assembly graphs using spacegraphcats reveals hidden sequence diversity 
bioRxiv preprint doi: https://doi.org/10.1101/2022.01.11.475838; this version posted January 17,2022 . The copyright holder for this preprint (which was not certified by peer review) is the author/funder, who has granted bioRxiv a license to display the preprint in perpetuity. It is made available under aCC-BY 4.0 International license.

CTitus Brown, Dominik Moritz, Michael P O’Brien, Felix Reidl, Taylor Reiter, Blair D Sullivan Genome Biology (2020-07-06) https://doi.org/d4bb

DOI: 10.1186/s13059-020-02066-4 ·PMID: 32631445 ·PMCID: PMC7336657

49. A General-Purpose Counting Filter

Prashant Pandey, Michael A Bender, Rob Johnson, Rob Patro Association for Computing Machinery (ACM) (2017-05-09) https://doi.org/gg29n9 DOI: $10.1145 / 3035918.3035963$

50. Centrifuge: rapid and sensitive classification of metagenomic sequences

Daehwan Kim, Li Song, Florian P Breitwieser, Steven L Salzberg

Genome Research (2016-12) https://doi.org/f9fnrr

DOI: 10.1101/gr.210641.116 ·PMID: 27852649 ·PMCID: PMC5131823

51. The rust language

Nicholas D Matsakis, Felix S Klock II

ACM SIGAda Ada Letters (2014-11-26) https://doi.org/gntjub

DOI: $10.1145 / 2692956.2663188$

52. An assembly and alignment-free method of phylogeny reconstruction from nextgeneration sequencing data

Huan Fan, Anthony R Ives, Yann Surget-Groba, Charles H Cannon

BMC Genomics (2015-07-14) https://doi.org/f7s6tp

DOI: 10.1186/s12864-015-1647-5 ·PMID: 26169061 · PMCID: PMC4501066

53. Welcome to sourmash! - sourmash 4.2.4.dev8+g1c46d7a5.d20220117 documentation https://sourmash.readthedocs.io/en/latest/

54. GitHub - luizirber/2020-cami: Preparing sourmash for CAMI 2 evaluations GitHub

https://github.com/luizirber/2020-cami

55. Minimap2: pairwise alignment for nucleotide sequences

Heng Li

Bioinformatics (2018-09-15) https://doi.org/gdhbqt

DOI: 10.1093/bioinformatics/bty191 · PMID: 29750242 · PMCID: PMC6137996

56. GitHub - dib-lab/genome-grist: map Illumina metagenomes to genomes!

GitHub

https://github.com/dib-lab/genome-grist

57. Sustainable data analysis with Snakemake

Felix Mölder, Kim Philipp Jablonski, Brice Letcher, Michael B Hall, Christopher H Tomkins-Tinch, Vanessa Sochat, Jan Forster, Soohyun Lee, Sven O Twardziok, Alexander Kanitz, ... Johannes Köster

F1000Research (2021-04-19) https://doi.org/gj76rq

DOI: 10.12688/f1000research.29032.2 · PMID: $\underline{34035898}$ • PMCID: PMC8114187

58. dib-lab/genome-grist: v0.7.4

CTitus Brown, Tessa Pierce Ward, Mohamed Abuelanin, Marisa Lim

Zenodo (2021-12-19) https://zenodo.org/record/5792144

59. Matplotlib: A 2D Graphics Environment

John D Hunter

Computing in Science \& Engineering (2007) https://doi.org/drbjhg

DOI: $10.1109 /$ mcse. 2007.55 
bioRxiv preprint doi: https://doi.org/10.1101/2022.01.11.475838; this version posted January $17,2022$. The copyright holder for this preprint (which was not certified by peer review) is the author/funder, who has granted bioRxiv a license to display the preprint in perpetuity. It is made available under aCC-BY 4.0 International license.

60. Jupyter Notebooks - a publishing format for reproducible computational workflows

Thomas Kluyver, Benjamin Ragan-Kelley, P\&\#233, Fernando Rez, Brian Granger, Matthias

Bussonnier, Jonathan Frederic, Kyle Kelley, Jessica Hamrick, Jason Grout, ... Jupyter

Development Team

Positioning and Power in Academic Publishing: Players, Agents and Agendas (2016)

https://ebooks.iospress.nl/doi/10.3233/978-1-61499-649-1-87

DOI: $10.3233 / 978-1-61499-649-1-87$

61. Array programming with NumPy

Charles R Harris, KJarrod Millman, Stéfan J van der Walt, Ralf Gommers, Pauli Virtanen, David Cournapeau, Eric Wieser, Julian Taylor, Sebastian Berg, Nathaniel J Smith, ... Travis E Oliphant Nature (2020-09-16) https://doi.org/ghbzf2

DOI: 10.1038/s41586-020-2649-2 ·PMID: $\underline{32939066}$ • PMCID: PMC7759461

62. pandas-dev/pandas: Pandas 1.4.0rc0

Jeff Reback, jbrockmendel, Wes McKinney, Joris Van den Bossche, Tom Augspurger, Phillip Cloud, Simon Hawkins, Matthew Roeschke, gfyoung, Sinhrks, ... Skipper Seabold

Zenodo (2022-01-06) https://zenodo.org/record/5824773

63. The Sequence Alignment/Map format and SAMtools

H Li, B Handsaker, A Wysoker, T Fennell, J Ruan, N Homer, G Marth, G Abecasis, R Durbin

Bioinformatics (2009-06-08) https://doi.org/ff6426

DOI: 10.1093/bioinformatics/btp352 · PMID: 19505943 · PMCID: PMC2723002

64. BEDTools: a flexible suite of utilities for comparing genomic features

Aaron R Quinlan, Ira M Hall

Bioinformatics (2010-03-15) https://doi.org/cmrms3

DOI: 10.1093/bioinformatics/btq033 · PMID: 20110278 • PMCID: PMC2832824

65. fastp: an ultra-fast all-in-one FASTQ preprocessor

Shifu Chen, Yanqing Zhou, Yaru Chen, Jia Gu

Bioinformatics (2018-09-01) https://doi.org/gd9mrb

DOI: 10.1093/bioinformatics/bty560 · PMID: 30423086 • PMCID: PMC6129281

66. khmer release v2.1: software for biological sequence analysis

Daniel Standage, Ali yari, Lisa J. Cohen, Michael R. Crusoe, Tim Head, Luiz Irber, Shannon EK Joslin, N B. Kingsley, Kevin D. Murray, Russell Neches, ... C Titus Brown

The Journal of Open Source Software (2017-07-03) https://doi.org/gntbpv

DOI: $10.21105 / j$ joss.00272

67. screed - short read sequence utils - screed $\mathbf{1 . 0}$ documentation

https://screed.readthedocs.io/en/v1.0/

68. GitHub - Ih3/seqtk: Toolkit for processing sequences in FASTA/Q formats

GitHub

https://github.com/lh3/seqtk

69. GitHub - ncbi/sra-tools: SRA Tools

GitHub

https://github.com/ncbi/sra-tools

70. Anaconda Documentation - Anaconda documentation https://docs.anaconda.com/

71. Bioconda: sustainable and comprehensive software distribution for the life sciences Björn Grüning, Ryan Dale, Andreas Sjödin, Brad A Chapman, Jillian Rowe, Christopher H Tomkins-Tinch, Renan Valieris, Johannes Köster, The Bioconda Team 
bioRxiv preprint doi: https://doi.org/10.1101/2022.01.11.475838; this version posted January 17,2022 . The copyright holder for this

preprint (which was not certified by peer review) is the author/funder, who has granted bioRxiv a license to display the preprint in perpetuity. It is made available under aCC-BY 4.0 International license.

Nature Methods (2018-07-02) https://doi.org/gd2xzp

DOI: 10.1038/s41592-018-0046-7 · PMID: 29967506

72. dib-lab/2021-paper-sourmash-gather-pipeline: v0.1

CTitus Brown

Zenodo (2021-12-20) https://zenodo.org/record/5793387

\section{Appendix}

1. In our current implementation in sourmash, when equivalent matches are available for a given rank, a match is chosen at random. This is an implementation decision that is not intrinsic to the algorithm itself.ఱ 